\title{
Review Article \\ Effects of Light Intensity Activity on CVD Risk Factors: A Systematic Review of Intervention Studies
}

\author{
Romeo B. Batacan Jr., ${ }^{1,2}$ Mitch J. Duncan, ${ }^{3}$ Vincent J. Dalbo, ${ }^{1,4}$ \\ Patrick S. Tucker, ${ }^{1,4}$ and Andrew S. Fenning ${ }^{1,2}$ \\ ${ }^{1}$ Institute for Health and Social Science Research, Central Queensland University, Bruce Highway, Rockhampton, QLD 4702, Australia \\ ${ }^{2}$ Centre for Physical Activity Studies, Central Queensland University, Bruce Highway, Rockhampton, QLD 4702, Australia \\ ${ }^{3}$ School of Medicine \& Public Health, Priority Research Centre for Physical Activity and Nutrition, Faculty of Health and Medicine, \\ The University of Newcastle, University Drive, Callaghan, NSW 2308, Australia \\ ${ }^{4}$ Clinical Biochemistry Laboratory, Central Queensland University, Bruce Highway, Rockhampton, QLD 4702, Australia
}

Correspondence should be addressed to Romeo B. Batacan Jr.; r.j.batacan@cqu.edu.au

Received 22 May 2015; Revised 13 September 2015; Accepted 20 September 2015

Academic Editor: Antonio Crisafulli

Copyright (C) 2015 Romeo B. Batacan Jr. et al. This is an open access article distributed under the Creative Commons Attribution License, which permits unrestricted use, distribution, and reproduction in any medium, provided the original work is properly cited.

\begin{abstract}
The effects of light intensity physical activity (LIPA) on cardiovascular disease (CVD) risk factors remain to be established. This review summarizes the effects of LIPA on CVD risk factors and CVD-related markers in adults. A systematic search of four electronic databases (PubMed, Academic Search Complete, SPORTDiscus, and CINAHL) examining LIPA and CVD risk factors (body composition, blood pressure, glucose, insulin, glycosylated hemoglobin, and lipid profile) and CVD-related markers (maximal oxygen uptake, heart rate, C-reactive protein, interleukin-6, tumor necrosis factor-alpha, and tumor necrosis factor receptors 1 and 2) published between 1970 and 2015 was performed on 15 March 2015. A total of 33 intervention studies examining the effect of LIPA on CVD risk factors and markers were included in this review. Results indicated that LIPA did not improve CVD risk factors and CVD-related markers in healthy individuals. LIPA was found to improve systolic and diastolic blood pressure in physically inactive populations with a medical condition. Reviewed studies show little support for the role of LIPA to reduce CVD risk factors. Many of the included studies were of low to fair study quality and used low doses of LIPA. Further studies are needed to establish the value of LIPA in reducing CVD risk.
\end{abstract}

\section{Introduction}

Cardiovascular disease (CVD) remains the leading cause of death worldwide [1]. Several biological risk factors, such as male gender, family history of heart disease, high blood pressure (BP), dyslipidemia, obesity, glucose abnormalities, insulin resistance, and lifestyle risk factors, such as smoking, poor diet, lack of physical activity, low cardiorespiratory fitness, excessive alcohol use, and stress, are associated with the development and progression of CVD $[2,3]$. Notably, these lifestyle risk factors strongly influence the established biological CVD risk factors and also affect novel pathways of risk such as inflammation [4]. For instance, physical activity and cardiorespiratory fitness (measured by maximal oxygen consumption $\left(\mathrm{VO}_{2} \max \right)$ and heart rate (HR)) are known to improve a number of traditional biological risk factors for CVD, including BP [5], high-density lipoprotein (HDL) cholesterol [6], body fat [7], and novel risk factors such as Creactive protein (CRP) levels [8].

There is excellent evidence that physical activity, particularly moderate-to-vigorous intensity physical activity (MVPA), is effective in the prevention and treatment of CVD $[9,10]$. The existing public health guidelines emphasize participation in MVPA to achieve health benefits $[9,10]$. However, the view that physical activity has to be moderate to vigorous to achieve cardiovascular risk reduction has been questioned [11]. It is suggested that physical activity performed at light intensity level can also provide health benefits $[12,13]$. As such, although early studies demonstrate that light intensity physical activity (LIPA) $\left(20<40 \% \mathrm{VO}_{2} \max\right.$ [14] $)$ 


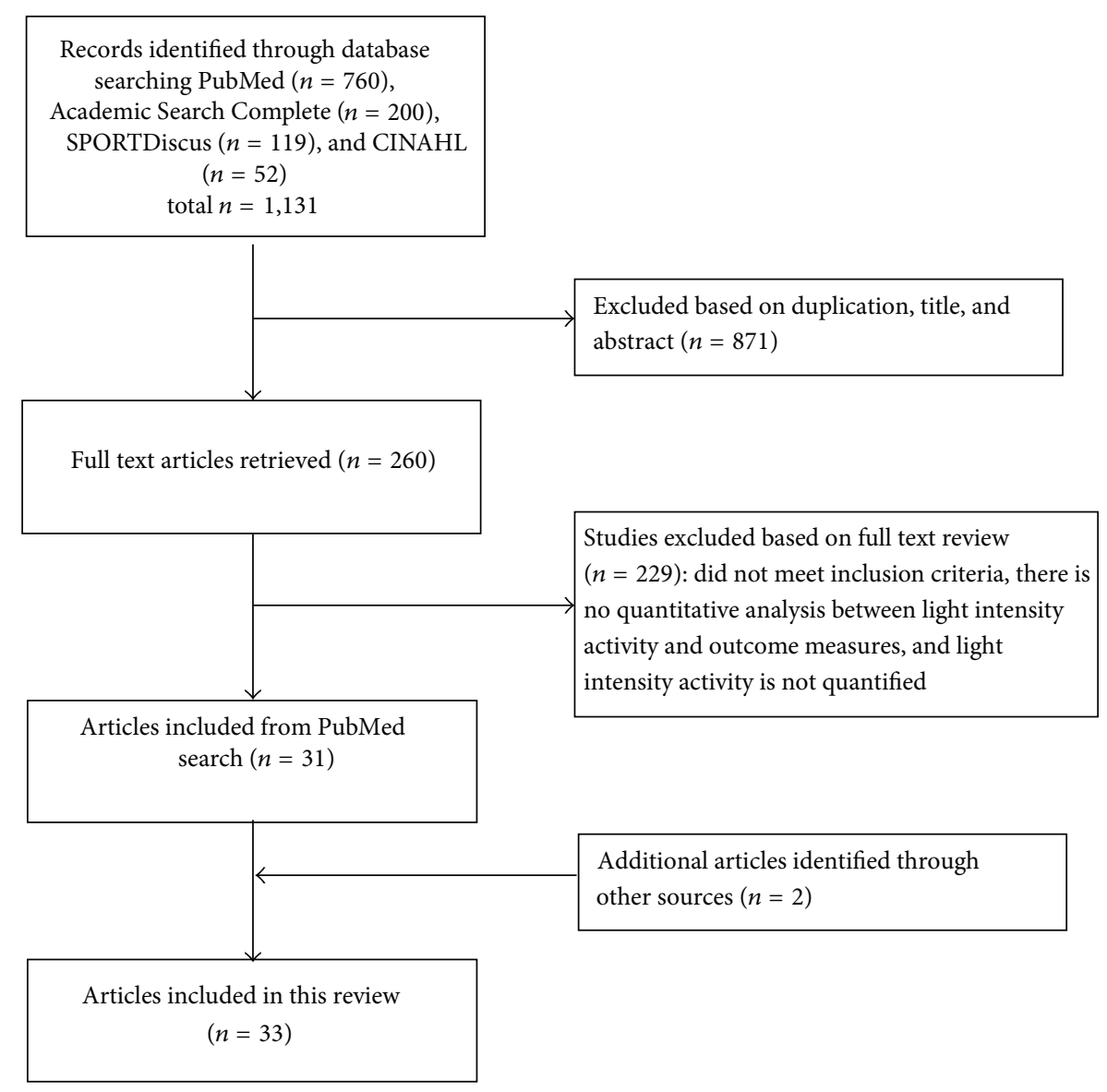

FIGURE 1: Flow diagram of study selection.

is not associated with reduced CVD and overall mortality rates $[15,16]$, there is growing recognition of the potential for LIPA to reduce disease risk, particularly CVD [17]. This is emphasized by cross-sectional studies demonstrating that LIPA is associated with CVD risk factors [12, 13, 18]. LIPA is important to understand, from a health perspective, as adults tend to spend a greater portion of their day $(6.5 \mathrm{hr} /$ day $[13,14])$ performing LIPA compared to MVPA $(0.7 \mathrm{hr} /$ day $[13,14])$. Many people often find it more attractive and attainable to perform LIPA than MVPA $\left(40<85 \% \mathrm{VO}_{2} \max \right)$ [19]. Furthermore, recent evidence suggests that muscle fiber recruitment during LIPA may potentially produce cellular signals which may regulate risk factors for disease [20].

As a result, clarifying the role of LIPA in CVD prevention is important given the amount of time people spend engaged in light intensity activities and its potential as an intervention target. To date, there has been no comprehensive review of literature describing the role of LIPA on CVD risk factors. Therefore, the aim of this review is to systematically examine the effects of LIPA on CVD risk factors (body composition, BP, glucose, insulin, glycosylated hemoglobin, total cholesterol, low-density lipoprotein (LDL) cholesterol, HDL cholesterol, and triglycerides) and other CVD-related markers $\left(\mathrm{VO}_{2}\right.$ max, $\mathrm{HR}, \mathrm{CRP}$, interleukin-6, tumor necrosis factor- (TNF-) alpha, TNF receptor 1 (TNFR1), and TNF receptor 2 (TNFR2)) in adults.

\section{Methods}

A systematic search was performed on 15 March 2015 according to PRISMA guidelines [21]. Articles were retrieved from PubMed, Academic Search Complete, SPORTDiscus, and CINAHL using multiple search criteria provided in Supplementary Table 1 in Supplementary Material available online at http://dx.doi.org/10.1155/2015/596367. Initially, titles and abstracts of identified articles were checked for relevance by two reviewers (RB and PT). Subsequently, both reviewers independently reviewed the full text of potentially eligible papers. Any disagreement between the two reviewers for inclusion was resolved through discussion. Additional articles were identified via hand-searching and reviewing the reference lists of relevant papers. Figure 1 presents the flow of papers through the study selection process.

Studies were considered to be eligible for inclusion based on the following criteria: (i) participants were $\geq 18$ years of age; (ii) the study examined at least one of the following CVD risk factors/markers in humans: body mass, body mass index (BMI), waist circumference (WC), hip circumference, waist-to-hip ratio (WHR), \% body fat, $\mathrm{HR}, \mathrm{BP}, \mathrm{VO}_{2}$ max, glucose (fasting or postprandial), glycosylated hemoglobin, insulin, total cholesterol, HDL cholesterol, LDL cholesterol, triglycerides, CRP, interleukin-6, TNF-alpha, TNF receptor 1, or TNF receptor 2 levels; (iii) the study reported 
an intervention (both randomized and nonrandomized) that imposed on participants a single or periodic bouts of LIPA defined as activities between $1.6<3.0$ METs, $20<40 \% \mathrm{VO}_{2}$ $\max$, and $20<40 \%$ heart rate reserve (HRR) or the relative intensity of $40<55 \%$ HR $\max [14,22]$; (iv) the study included quantitative analysis (statistical comparison of intervention to baseline or a control group) of the effect of LIPA on at least one of the outcome measures; (v) the study was published or accepted for publication in refereed journals from 1970 up to and including the search date; (vi) the study was published in the English language. Due to the lack of a standardized definition of LIPA for resistance training, only aerobic/flexibility exercises were included in the study.

Two authors (RB and PT) independently assessed the quality of the studies that met the inclusion criteria (Table 1). The risk of bias and strength of evidence from individual studies were assessed using Downs and Black Checklist [23], allowing for the assessment of the methodological quality of randomized controlled trials and nonrandomized studies of health care interventions. This 27-point checklist assesses the strength of reporting, external validity, internal validity, and statistical power. As some questions are worth more than one point, the maximum score that can be received is 32. Adapted from another systematic review [24], the score obtained by each study was divided by 32 and multiplied by 100 to provide a "study quality percentage." Study quality percentages were then classified as high (66.7\% or higher), fair (between 50.0 and 66.6\%), and low (less than 50.0\%) [24].

Following data extraction, the interventions included in this review were heterogeneous in terms of the type, frequency, and duration of physical activities, as well as body mass, physical fitness, and dietary intake of the participants. Thus, meta-analyses or pooling of data across studies would be inappropriate so a qualitative synthesis of the evidence was performed instead.

A modified form of coding system described by Sallis et al. [25] was used to summarize the effect of LIPA on CVD risk factors/markers. If $0-33 \%$ of the studies reported a statistically significant difference between LIPA and CVD risk factors/markers, the result was categorized as no effect (0). If $34-59 \%$ of the studies reported a statistically significant difference, the result was categorized as inconsistent (?). If $60-100 \%$ of the studies reported a statistically significant difference, the result was rated as positive $(+)$ or negative $(-)$, respective of the direction of the effect. When four or more studies supported a difference or no difference, it was coded as,++-- , or 00 to indicate consistent observations. The ?? code indicated a marker that has been examined in four or more studies with inconsistent findings (e.g., out of 5 studies, 3 indicated a significant positive effect and 2 indicated a significant negative effect).

Results were then stratified by health status of the population (healthy or those with a medical condition). Studies in which participant physical activity was less than $150 \mathrm{~min} / \mathrm{wk}$ of moderate intensity physical activity or $75 \mathrm{~min} / \mathrm{wk}$ of vigorous intensity physical activity or participants were not engaged in regular physical activity/exercise (as described in the primary study) or participants were defined as sedentary were subsequently classified as "physically inactive" and the results are summarized separately for these studies.

\section{Results}

General study characteristics are summarized in Table 1; more detailed study characteristics are presented in Supplementary Table 2. All studies had LIPA intervention with the number of study participants in the LIPA group ranging from $n=6-39$. Participants were primarily young, adult, and males (18-39 years old). Duration of LIPA interventions ranged from an acute bout of training lasting 5 minutes to chronic training lasting $30 \mathrm{~min}$ per session, 3 times per week, for 9 months. The exercise modalities involved stretching and calisthenics $(n=1)$, hand rim wheelchair training $(n=1)$, yoga $(n=1)$, standing $(n=2)$, slow flexibility exercises $(n=3)$, stationary cycling $(n=7)$, and slow home/outdoor or treadmill walking $(n=18)$. Fifteen [26-40] of the 33 reviewed studies had participants classified as overweight or obese and 7 studies [31, 33, 34, 36, 41-43] consisted of participants suffering from medical conditions (hypertension, diabetes mellitus, chronic heart failure, gestational diabetes, colorectal cancer, metabolic syndrome, and HIV infection).

Studies included in this review were assigned a quality rating. The study quality was rated low in 11 studies, fair in 20 studies, and high in 2 studies. Over $90 \%$ of the studies scored 1 point for defining study objectives, describing exposure and outcome variables, utilizing random sampling of the target population, providing data sources, and describing the data collection process. Seventeen studies (51\%) [26, 34, 36, 39, 42, 44-55] did not describe participant recruitment methods and 10 studies (30\%) [26, 40, 44, 46-52] did not report inclusion criteria. Five studies (15\%) [33, 34, 41, 56] did not report compliance rates. Of the studies that reported compliance, compliance was generally good with participants completing $81 \%$ to $100 \%$ of the activity sessions implemented per study design. Six studies [26, 31, 35, 38-40] reported power calculations relevant to their study aims. All but 4 studies [26, 30, 31, 33] performed the activity sessions in a laboratory/clinic directly supervised by a research staff. Those not conducted in the laboratory, light intensity activity was performed outdoors/at home with participants monitoring their own HR.

A summary table of the effect of LIPA on CVD risk factors and markers can be found in Table 2; the effects of LIPA on CVD risk factors/markers reported in each study are presented in Supplementary Table 3. Results demonstrated LIPA training interventions to have no significant effect on markers of body composition in physically inactive or healthy, with a medical condition, adults. All studies that examined the effect of LIPA on body mass [26, 29, 35, 53], WC [31, 32, 35, 41], BMI [26, 31, 32, 41], and \% body fat $[30,32]$ reported no significant change. LIPA was found to have no effect on systolic or diastolic BP in healthy adults while improvements in BP were found in physically inactive populations with a medical condition. Three [31, 32, 39] of 9 studies (33\%) reported significant decreases in systolic BP while 2 [31, 39] of 9 studies (22\%) reported significant decreases in diastolic BP. 


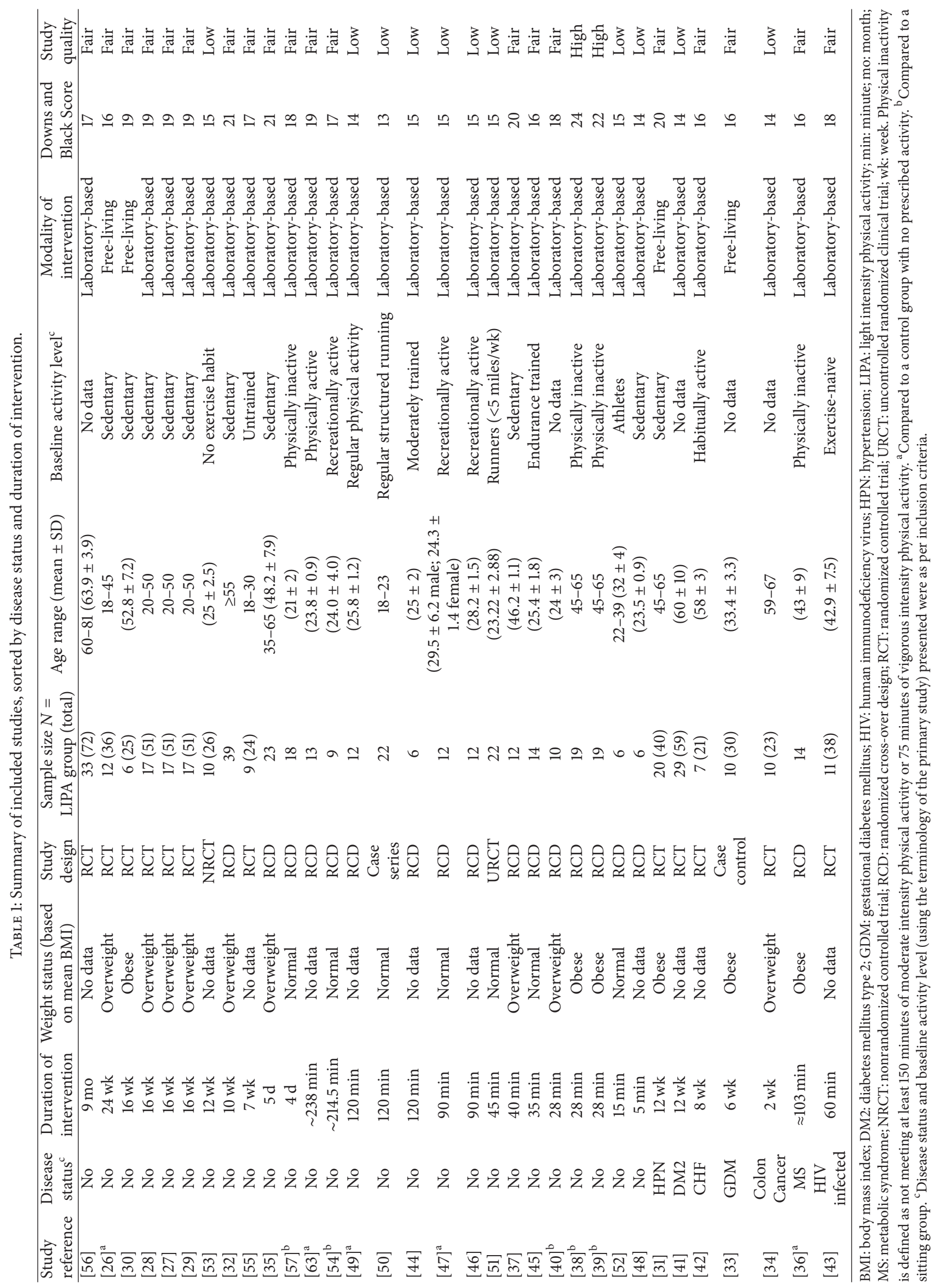


TABLE 2: Summary of studies examining the effect of LIPA on CVD risk factors and CVD-related markers.

\begin{tabular}{|c|c|c|c|c|c|c|c|c|}
\hline \multirow{2}{*}{$\begin{array}{l}\text { Marker } \\
\text { CVD risk factors }\end{array}$} & \multicolumn{2}{|c|}{$\begin{array}{l}\text { Summary coding of studies } \\
\text { involving physically } \\
\text { inactive population }\end{array}$} & \multicolumn{2}{|c|}{$\begin{array}{l}\text { Summary coding of studies } \\
\text { involving populations with } \\
\text { a medical condition }\end{array}$} & \multicolumn{2}{|c|}{$\begin{array}{c}\text { Summary coding of studies } \\
\text { involving healthy } \\
\text { population }\end{array}$} & \multicolumn{2}{|c|}{$\begin{array}{l}\text { Summary coding of all } \\
\text { included studies }\end{array}$} \\
\hline & $\begin{array}{c}n / N \\
(\%)^{a, b, c}\end{array}$ & $\begin{array}{c}\text { Effect } \\
(0 /-/+/ \text { ? })^{\mathrm{d}}\end{array}$ & $\begin{array}{c}n / N \\
(\%)^{a, b, c}\end{array}$ & $\begin{array}{c}\text { Effect } \\
(0 /-/+/ \text { ? })^{\mathrm{d}}\end{array}$ & $\begin{array}{c}n / N \\
(\%)^{a, b, c}\end{array}$ & $\begin{array}{c}\text { Effect } \\
(0 /-/+/ ?)^{\mathrm{d}}\end{array}$ & $n / N(\%)^{\mathrm{a}, \mathrm{b}, \mathrm{c}}$ & $\begin{array}{c}\text { Effect } \\
(0 /-/+/ ?)^{\mathrm{d}}\end{array}$ \\
\hline Body mass & $0 / 4(0 \%)$ & No (00) & $0 / 0(0 \%)$ & NA & $0 / 4(0 \%)$ & No (00) & $0 / 5(0 \%)$ & No (00) \\
\hline WC & $0 / 3(0 \%)$ & No $(0)$ & $0 / 2(0 \%)$ & No $(0)$ & $0 / 0(0 \%)$ & NA & $0 / 4(0 \%)$ & No $(00)$ \\
\hline BMI & $0 / 3(0 \%)$ & No $(0)$ & $0 / 2(0 \%)$ & No $(0)$ & $0 / 0(0 \%)$ & NA & $0 / 4(0 \%)$ & No $(00)$ \\
\hline$\% \mathrm{BF}$ & $0 / 2(0 \%)$ & No $(0)$ & $0 / 0(0 \%)$ & NA & $0 / 0(0 \%)$ & NA & $0 / 2(0 \%)$ & No $(0)$ \\
\hline Systolic BP & $3 / 5(60 \%)$ & Positive $(++)$ & $2 / 3(67 \%)$ & Positive (+) & $0 / 5(0 \%)$ & No $(00)$ & $3 / 9(33 \%)$ & No $(00)$ \\
\hline Diastolic BP & $2 / 5(40 \%)$ & $\begin{array}{c}\text { Inconsistent } \\
\text { (??) }\end{array}$ & $2 / 3(67 \%)$ & Positive (+) & $0 / 5(0 \%)$ & No $(00)$ & $2 / 9(22 \%)$ & No $(00)$ \\
\hline Glucose & $1 / 8(12 \%)$ & No $(00)$ & $1 / 3(33 \%)$ & No $(0)$ & $0 / 8(0 \%)$ & No $(00)$ & $3 / 16(19 \%)$ & No $(00)$ \\
\hline Insulin & $1 / 8(12 \%)$ & No $(00)$ & $0 / 1(0 \%)$ & NA & $0 / 8(0 \%)$ & No $(00)$ & $1 / 13(8 \%)$ & No $(00)$ \\
\hline HbalC & $0 / 1(0 \%)$ & NA & $0 / 1(0 \%)$ & NA & $0 / 0(0 \%)$ & NA & $0 / 2(0 \%)$ & No $(0)$ \\
\hline Total cholesterol & $1 / 5(20 \%)$ & No $(00)$ & $0 / 0(0 \%)$ & NA & $1 / 6(17 \%)$ & No $(00)$ & $2 / 11(18 \%)$ & No $(00)$ \\
\hline HDL cholesterol & $0 / 7(0 \%)$ & No $(00)$ & $0 / 1(0 \%)$ & NA & $1 / 8(12 \%)$ & No $(00)$ & $1 / 13(8 \%)$ & No $(00)$ \\
\hline LDL cholesterol & $0 / 4(0 \%)$ & No $(00)$ & $0 / 0(0 \%)$ & NA & $0 / 3(0 \%)$ & No $(0)$ & $0 / 6(0 \%)$ & No $(00)$ \\
\hline Triglycerides & $2 / 8(25 \%)$ & No $(00)$ & $0 / 1(0 \%)$ & NA & $4 / 7$ (57\%) & $\begin{array}{c}\text { Inconsistent } \\
\text { (??) }\end{array}$ & $5 / 13(38 \%)$ & $\begin{array}{c}\text { Inconsistent } \\
\text { (??) }\end{array}$ \\
\hline CVD-related markers & $\begin{array}{c}n / N \\
(\%)^{\mathrm{a}, \mathrm{b}, \mathrm{c}}\end{array}$ & $\begin{array}{c}\text { Effect } \\
(0 /-/+/ ?)^{\mathrm{d}} \\
\end{array}$ & $\begin{array}{c}n / N \\
(\%)^{\mathrm{a}, \mathrm{b}, \mathrm{c}}\end{array}$ & $\begin{array}{c}\text { Effect } \\
(0 /-/+/ ?)^{\mathrm{d}}\end{array}$ & $\begin{array}{c}n / N \\
(\%)^{\mathrm{a}, \mathrm{b}, \mathrm{c}}\end{array}$ & $\begin{array}{c}\text { Effect } \\
(0 /-/+/ ?)^{\mathrm{d}}\end{array}$ & $n / N(\%)^{\mathrm{a}, \mathrm{b}, \mathrm{c}}$ & $\begin{array}{c}\text { Effect } \\
(0 /-/+/ ?)^{\mathrm{d}}\end{array}$ \\
\hline $\mathrm{VO}_{2} \max$ & $2 / 5(40 \%)$ & $\begin{array}{c}\text { Inconsistent } \\
(? ?)\end{array}$ & $0 / 1(0 \%)$ & NA & $1 / 2(50 \%)$ & $\begin{array}{c}\text { Inconsistent } \\
(?)\end{array}$ & $3 / 8(38 \%)$ & $\begin{array}{c}\text { Inconsistent } \\
\text { (??) }\end{array}$ \\
\hline Resting HR & $1 / 4(25 \%)$ & No $(00)$ & $0 / 1(0 \%)$ & NA & $0 / 3(0 \%)$ & No $(0)$ & $1 / 5(20 \%)$ & No $(00)$ \\
\hline CRP & $0 / 2(0 \%)$ & No $(0)$ & $0 / 0(0 \%)$ & NA & $0 / 0(0 \%)$ & NA & $0 / 2(0 \%)$ & No $(0)$ \\
\hline Interleukin-6 & $0 / 3(0 \%)$ & No $(0)$ & $0 / 2(0 \%)$ & No $(0 \%)$ & $0 / 0(0 \%)$ & NA & $0 / 4(0 \%)$ & No $(00)$ \\
\hline TNF-alpha & $0 / 1(0 \%)$ & NA & $0 / 1(0 \%)$ & NA & $0 / 0(0 \%)$ & NA & $0 / 2(0 \%)$ & No $(0)$ \\
\hline
\end{tabular}

${ }^{a} n=$ number of studies reporting difference in the expected direction.

${ }^{\mathrm{b}} \mathrm{N}=$ number of identified studies of interest.

${ }^{c}(\%)=$ percentage of studies reporting differences in the expected direction.

${ }^{\mathrm{d}}$ Summary effect. No effect (0): 0-33\% of studies reported significant differences; inconsistent (?): $34-59 \%$ of studies reported significant differences; positive $(+)$ or negative (-) effect: $60-100 \%$ of studies demonstrated significant differences; $\geq 4$ studies: positive (++), negative (--), no effect (00), and inconsistent findings (??).

CVD markers (waist-to-hip ratio, heart rate maximal, and tumor necrosis factor receptor 2) with only one study demonstrating the effect of light intensity activity were excluded in this summary table.

BF: body fat; BMI: body mass index; BP: blood pressure; CRP: C-reactive protein; CVD: cardiovascular disease; Hbalc: glycosylated hemoglobin; HDL: highdensity lipoprotein; HR: heart rate; LDL; low-intensity lipoprotein; NA: not applicable; TNF: tumor necrosis factor; $\mathrm{VO}_{2}$ max: maximal oxygen uptake; WC: waist circumference.

LIPA was found to have no effect on glucose and insulin response in physically inactive or healthy, with a medical condition, adults. Three $[33,38,40]$ of 16 studies (19\%) reported significant decreases in glucose and 1 of $13(8 \%)$ reported significant decrease in insulin level. When the effect of LIPA on blood lipid markers was examined, no significant changes were found for total cholesterol, HDL cholesterol, LDL cholesterol, or triglycerides in physically inactive or healthy individuals and inconsistent findings on triglycerides in healthy adults. One [50] of 11 studies (9\%) reported a significant increase in total cholesterol while $2[26,46]$ of 11 studies (18\%) reported significant decreases in total cholesterol. One [50] of 13 studies (8\%) reported a significant increase in HDL cholesterol, 5 [36, 46, 49, 54, 57] of 13 studies (38\%) reported significant increases in triglycerides, and 0 of 6 studies (0\%) reported an effect on LDL cholesterol.
Regarding other CVD-related markers, the effect of LIPA on $\mathrm{VO}_{2}$ max is inconclusive in physically inactive or healthy adults. Three $[27,32,56]$ of 8 studies (38\%) reported significant increases in $\mathrm{VO}_{2}$ max. LIPA was also found to have no effect on resting HR in physically inactive or healthy adults. One [32] of 5 studies (20\%) reported a significant reduction in resting HR. All studies that examined the effect of LIPA on CRP [30, 37], interleukin- 6 [30, 34, 37, 43], and TNF-alpha $[30,34]$ reported no significant effect in physically inactive, with a medical condition, adults.

\section{Discussion}

The effect of LIPA on markers of cardiovascular risk factors was systematically reviewed. LIPA resulted in no significant improvements in body composition, glucose, insulin 
(in physically inactive or healthy, with a medical condition, adults), total cholesterol, HDL cholesterol, LDL cholesterol (in physically inactive or healthy adults), or triglycerides (in physically inactive adults) and inconsistent findings on triglycerides in healthy adults. On the other hand, LIPA was found to improve systolic and diastolic BP in physically inactive populations with a medical condition. Additionally, when examining CVD-related markers, we found inconsistent results regarding the effect of LIPA on $\mathrm{VO}_{2}$ max in physically inactive or healthy adults, no significant changes on resting $\mathrm{HR}$ in physically inactive or healthy adults, and no significant changes on inflammatory markers in physically inactive or with a medical condition adults.

Nine studies [26, 29-32, 35, 41, 53, 55] examined the effect of LIPA on body composition and found no effect in either physically inactive or healthy, with a medical condition, populations. One study concluded that LIPA performed $30 \mathrm{~min}, 8$ times a day, for 5 days, did not result in any significant change on body mass and WC [35]. The rest of the studies demonstrated that LIPA performed $30-90 \mathrm{~min}, 3$ to 5 times per wk, for $\geq 7 \mathrm{wk}$, did not result in any significant effect on body mass $[26,29,53,55]$, WC $[31,32,41]$, BMI $[26,31,32,41]$, WHR [26], or \% body fat [30,32]. This result is consistent with previous research findings that conclude at least $250 \mathrm{~min} /$ wk of moderate intensity ( $\geq 3 \mathrm{METs}$ ) training is needed if the primary purpose of the training program is to elicit reductions in body mass and fat mass $[58,59]$. There are no recommended durations of LIPA required to elicit weight loss; however, the amount of LIPA required to improve body composition is likely to be much greater than that required for MVPA given the reduced intensity level. Physical activity alone if greater than $250 \mathrm{~min} / \mathrm{wk}$ without caloric restriction has a limited influence on body composition $[27,28,60]$ and may only cause $1-3 \%$ change in body mass and adipose tissue [61]. In addition, evidence suggests that the total volume of physical activity is a key factor in achieving weight loss [62]. An individual intending to lose weight through physical activity without dietary restriction would need to engage in a large volume (26 MET-hr per wk) of physical activity to achieve a $5 \%$ weight reduction [62]. In most studies to date, the volume of LIPA used is less ( $<10.5$ MET-hr per wk) than the $26 \mathrm{MET}-\mathrm{hr}$ per wk that may be required to improve body composition.

Findings of this review also indicate no significant changes in resting BP in healthy adults but found significant improvements in physically inactive individuals with a medical condition. Participants who followed a single bout (15 min) [52] and periodic bouts (2 min every $20 \mathrm{~min}$ over $5 \mathrm{hr}$ period) [40] of treadmill walking, and long term (30$60 \mathrm{~min} 3-5 \mathrm{x} / \mathrm{wk}, \geq 10 \mathrm{wk})$ LIPA $[30,32,41,53,56]$ demonstrated no significant changes in resting BP. Two studies $[31,32]$ reported a decrease in systolic BP and one study reported [31] a decrease in diastolic BP following $\geq 10 \mathrm{wk}$ of walking [31] and a combination of treadmill walking, stationary cycling and stepping [32]. In both studies, the improved BP response was found in physically inactive participants with hypertension. Similarly, in prehypertensive and hypertensive physically inactive, obese adults, one study [39] with a different study design (randomized cross-over study breaking up prolonged sitting with LIPA breaks) found significant reductions in systolic and diastolic BP in individuals interrupting sitting time with light intensity walking relative to individuals with uninterrupted sitting. Thus, LIPA appears unlikely to influence the BP response in normotensive populations but may be able to provide an effect in hypertensive, physically inactive populations.

There were no significant improvements in glucose and insulin response following LIPA in either physically inactive or healthy, with a medical condition, adults. All 6 studies $[36,44,45,47,49,63]$ reported no effects of glucose and insulin response during a single bout (35-237.5 min) of LIPA. Following periodic bouts $(214.5 \pm 28 \mathrm{~min}$ divided in 9 bouts, $30-60 \mathrm{~min} 3 \mathrm{x} / \mathrm{wk}, 30 \mathrm{~min} 8 \mathrm{x} / \mathrm{day}, 4 \mathrm{hr}$ walking, and $2 \mathrm{hr}$ standing/day) of LIPA, 7 [30, 32, 35, 41, 53, 54, 57] of 10 studies reported no significant changes in glucose and $6[30,32,35$, $53,54,57]$ of 7 studies reported no significant changes in insulin. These results are consistent with epidemiological data showing no significant association between fasting glucose and time spent performing LIPA (5.7-6.0 hr/day) (but not with $2 \mathrm{hr}$ plasma glucose which was found to be significantly associated with LIPA) $[12,13]$. In contrast, 2 studies [38, 40] reported a decrease in postprandial glucose (and insulin [38]) after interrupting sitting with light intensity standing/walking. These laboratory-based studies compared a light intensity standing/walking group to a sitting group, employed LIPA (14 sessions of 2 min LIPA separated by 20 min sitting period) dispersed throughout the day, and measured postprandial glucose. These findings were validated in a recent meta-analysis that found significant reductions in blood glucose postprandial response and insulin levels after interrupting sedentary periods with LIPA breaks [64]. Another study [33] with a longer, structured, light intensity walking intervention period (120-160 min/wk walking for $6 \mathrm{wk}$ ) also demonstrated reductions in capillary glucose concentrations post-intervention compared to baseline. This study used obese women with gestational diabetes. In summary, there is no consistent intervention evidence to support improved glucose metabolism with LIPA in healthy adults. Studies $[33,38,40]$ suggesting that LIPA may improve glucose and insulin response examined individuals with higher glucose baseline values or compared LIPA to a sedentary (sitting) group or used multiple bouts of LIPA dispersed throughout the day. Thus, there may be some evidence to support the view that LIPA influences glucose and insulin metabolism, but this evidence appears to be limited to individuals (1) with impaired cardiometabolic function or (2) who are compared to no activity (sedentary) control groups.

Most studies in this review reported no significant change in total cholesterol $[30,32,40,47,49,51,53,57]$, HDL cholesterol $[26,30,32,36,40,46,47,49,51,53,57,63]$, LDL cholesterol $[26,30,32,46,51,57]$, in physically inactive or healthy individuals, or in triglycerides $[26,30,32,35$, $40,51,53,63$ ] in physically inactive adults following LIPA. Inconsistent findings were found on the effect of LIPA on triglycerides in healthy adults. This result is not consistent with evidence from epidemiological studies demonstrating a beneficial association of larger volumes of LIPA with HDL cholesterol (4.3 hr/day of LIPA) [65] and triglycerides 
(5.7-6.0 hr/day; < $150 \mathrm{~min} /$ wk MVPA, but LIPA exceeded sedentary behavior) $[18,66]$. Conversely, similar results were found in a recent meta-analysis that reported no significant reductions in triglycerides after interrupting sedentary periods with LIPA breaks [64]. These conflicting results reported in the literature in regard to the effect of LIPA in blood lipid markers may be due to differences in study protocol and participants' baseline lipid levels.

In this review, $5[36,46,49,54,57]$ of 10 studies demonstrated significant reductions in triglycerides following LIPA in healthy adults. These studies used short intervention periods ( $\leq 4$ days) of light intensity walking and 3 studies $[36,49,54]$ used a high fat test meal prior to blood sampling. This immediate lowering of serum triglycerides following LIPA is most likely due to enhanced triglyceride peripheral tissue uptake of serum triglycerides that result from exerciseinduced activity of lipoprotein lipase, the rate limiting enzyme for the hydrolysis of triglyceride-rich lipoproteins [67]. The increased activity of lipoprotein lipase (persisting up to $18 \mathrm{hr}$ ) following muscular contractions causes an increase in the removal of triglycerides from the circulation [68]. Unfortunately, none of these studies explored whether or not triglyceride reductions persisted for more than $24 \mathrm{hr}$ following LIPA bout.

$\mathrm{VO}_{2}$ max, resting $\mathrm{HR}$, and inflammatory markers that are known to impact CVD risk factors were also examined. LIPA had inconsistent results in regard to the effects on $\mathrm{VO}_{2}$ max and no effect on resting HR in physically inactive or healthy adults. Studies employing long term ( $\geq 8 \mathrm{wk}$ ) LIPA protocols generally reported no change $[28-30,42,48,53,56]$, while others reported improvement in $\mathrm{VO}_{2} \max [27,32,56]$ and HR [32]. It is possible that certain types of aerobic exercise may lead to health-related benefits and yet may not be of sufficient quantity or quality to improve $\mathrm{VO}_{2} \max$ or decrease resting HR [69]. Despres and Lamarche [70] proposed that prolonged (exact duration not specified) low intensity (approximately 50\% $\mathrm{VO}_{2} \max$ ) endurance exercise performed 45-60 min on an almost daily basis significantly improved insulin sensitivity and lipoprotein metabolism through mechanisms that are likely to be independent of the training-related changes in cardiorespiratory fitness. The proposed mechanisms included the net increase in energy expenditure and losses in total body fat and abdominal adipose tissue which contributed to improved carbohydrate and lipid metabolism [70]. This hypothesis, however, remains to be established. At present, only $3[27,32,56]$ of 7 studies reported a positive effect on $\mathrm{VO}_{2}$ max; $2[27,32]$ of these 3 studies examined physically inactive, overweight adults. The third study [56] neglected to report baseline physical activity and BMI. Thus, the beneficial effects of LIPA, in regard to adaptations to $\mathrm{VO}_{2}$ max, are equivocal and may be most pronounced in individuals with low levels of physical activity $[71,72]$ suggesting that the benefits of LIPA on $\mathrm{VO}_{2}$ max may be limited to populations who are least active.

No significant changes in inflammatory markers (CRP, interleukin-6, and TNF-alpha) were found in physically inactive or with a medical condition participants engaging in a single bout $(40-60 \mathrm{~min})[37,43]$, periodic bouts ( $40 \mathrm{~min} /$ day for two wk) [34], or long term (30 min 3x/wk for $16 \mathrm{wk}$ ) [30]
LIPA. Research in this area is limited and more studies are needed to clarify the effect of LIPA on inflammatory markers. Results from the interventions ( 3 out of 4 studies) included in this review demonstrate that acute effects are unlikely to occur and future research should seek to examine changes in inflammatory markers following participation in LIPA over longer time periods.

This review provides consistent evidence that LIPA is not effective at improving CVD risk factors and other CVDrelated health markers in apparently healthy individuals. Some evidence surfaced suggesting that LIPA may improve markers of CVD risk factors (BP) in physically inactive adults with a medical condition. These findings provide some support to cross-sectional studies suggesting that LIPA may be beneficial in elderly, physically inactive, with a medical condition, individuals $[65,73,74]$. However, due to limited intervention studies available that have examined these cohorts of individuals, it is difficult to make conclusions with full certainty. Future studies should attempt to elucidate the effects of LIPA in elderly, physically inactive, with a medical condition, adults. Since LIPA is low intensity and appears to be most practical in physically inactive populations, daily LIPA and MVPA of participants should be accounted for in future work.

The dose of LIPA used in the reviewed studies was modest in comparison to the volume of LIPA typically performed by individuals (e.g., $\leq 150 \mathrm{~min} / \mathrm{wk}$ which equates to $<10.5 \mathrm{MET}$ $\mathrm{hr}$ per wk). Therefore, future studies are encouraged to use greater doses (much higher than the recommended $150 \mathrm{~min} /$ wk moderate intensity physical activity due to the reduced intensity level of LIPA) to assist in clarifying the role of LIPA to elicit positive changes in CVD risk factors and CVD-related markers.

\section{Conclusions}

Although cross-sectional research findings $[12,13,18]$ suggest that LIPA may help to improve an individual's metabolic profile, there is no evidence to support the effect of LIPA in providing positive changes in CVD risk factors in healthy adults. Little intervention evidence was found to support the positive effect of LIPA in CVD risk factors in physically inactive adults with a medical condition. In particular, significant improvements in BP following LIPA were achieved by physically inactive, hypertensive individuals $[31,32,39]$. However, it should be noted that many studies reviewed did not control, either statistically or by design, for potential confounding variables such as controlling for accumulated MVPA or monitoring dietary intake. Most of the studies have also used small doses of LIPA ( $<10.5$ MET-hr per wk). Given that adults spend a considerable proportion of their day $(6.5 \mathrm{hr} /$ day $[13,14])$ performing LIPA, it may be possible that this volume of LIPA is not enough of a stimulus to promote favorable adaptations in the examined biological markers of CVD risk. Aside from increasing the volume, it may also be worthwhile to examine the effects of LIPA dispersed throughout the day similar to recent studies $[35,38-40]$ that have used regular short bouts of LIPA to interrupt prolonged 
periods of sitting. This may be useful as recent meta-analysis found these breaks in sitting to be associated with improved glucose and insulin response [64]. In summary, there may be some evidence to support the view that LIPA influences some CVD risk factors in certain populations, but more welldesigned experiments with greater control of confounding factors are required to confirm this.

\section{Conflict of Interests}

The authors declare that there is no conflict of interests regarding the publication of this paper.

\section{Acknowledgments}

R. B. Batacan is supported by the Strategic Research Scholarship grant from Central Queensland University. This paper is in part supported by CQUniversity Health Collaborative Research Network. Mitch J. Duncan is supported by a Future Leader Fellowship (ID 100029) from the National Heart Foundation of Australia.

\section{References}

[1] WHO, Global Status Report on Noncommunicable Diseases 2010, World Health Organization, Geneva, Switzerland, 2011.

[2] D. S. Prasad, Z. Kabir, A. K. Dash, and B. C. Das, "Cardiovascular risk factors in developing countries: a review of clinicoepidemiological evidence," CVD Prevention and Control, vol. 5, no. 4, pp. 115-123, 2010.

[3] M. R. Carnethon, S. S. Gidding, R. Nehgme, S. Sidney, D. R. Jacobs Jr., and K. Liu, "Cardiorespiratory fitness in young adulthood and the development of cardiovascular disease risk factors," The Journal of the American Medical Association, vol. 290, no. 23, pp. 3092-3100, 2003.

[4] D. Mozaffarian, P. W. F. Wilson, and W. B. Kannel, "Beyond established and novel risk factors: lifestyle risk factors for cardiovascular disease," Circulation, vol. 117, no. 23, pp. 30313038, 2008.

[5] S. P. Whelton, A. Chin, X. Xin, and J. He, "Effect of aerobic exercise on blood pressure: a meta-analysis of randomized, controlled trials," Annals of Internal Medicine, vol. 136, no. 7, pp. 493-503, 2002.

[6] J. L. Durstine, P. W. Grandjean, P. G. Davis, M. A. Ferguson, N. L. Alderson, and K. D. DuBose, "Blood lipid and lipoprotein adaptations to exercise: a quantitative analysis," Sports Medicine, vol. 31, no. 15, pp. 1033-1062, 2001.

[7] S. L. Wong, P. T. Katzmarzyk, M. Z. Nichaman, T. S. Church, S. N. Blair, and R. Ross, "Cardiorespiratory fitness is associated with lower abdominal fat independent of body mass index," Medicine and Science in Sports and Exercise, vol. 36, no. 2, pp. 286-291, 2004.

[8] D. Aronson, R. Sella, M. Sheikh-Ahmad et al., "The association between cardiorespiratory fitness and C-reactive protein in subjects with the metabolic syndrome," Journal of the American College of Cardiology, vol. 44, no. 10, pp. 2003-2007, 2004.

[9] P. D. Thompson, D. Buchner, I. L. Piña et al., "Exercise and physical activity in the prevention and treatment of atherosclerotic cardiovascular disease: a statement from the council on clinical cardiology (subcommittee on exercise, rehabilitation, and prevention) and the council on nutrition, physical activity, and metabolism (subcommittee on physical activity)," Circulation, vol. 107, no. 24, pp. 3109-3116, 2003.

[10] G. A. Gaesser, "Exercise for prevention and treatment of cardiovascular disease, type 2 diabetes, and metabolic syndrome," Current Diabetes Reports, vol. 7, no. 1, pp. 14-19, 2007.

[11] S. G. Wannamethee and A. G. Shaper, "Physical activity in the prevention of cardiovascular disease: an epidemiological perspective," Sports Medicine, vol. 31, no. 2, pp. 101-114, 2001.

[12] G. N. Healy, D. W. Dunstan, J. Salmon, E. Cerin, J. E. Shaw, and N. Owen, "Objectively measured light-intensity physical activity is independently associated with 2-h plasma glucose," Diabetes Care, vol. 30, no. 6, pp. 1384-1389, 2007.

[13] G. N. Healy, K. Wijndaele, D. W. Dunstan, J. E. Shaw, J. Salmon, and N. Owen, "Objectively measured sedentary time, physical activity, and metabolic risk the Australian Diabetes, Obesity and Lifestyle Study (AusDiab)," Diabetes Care, vol. 31, no. 2, pp. 369371, 2008.

[14] K. Norton, L. Norton, and D. Sadgrove, "Position statement on physical activity and exercise intensity terminology," Journal of Science and Medicine in Sport, vol. 13, no. 5, pp. 496-502, 2010.

[15] I.-M. Lee and R. S. Paffenbarger Jr., "Associations of light, moderate, and vigorous intensity physical activity with longevity: the Harvard Alumni Health Study," American Journal of Epidemiology, vol. 151, no. 3, pp. 293-299, 2000.

[16] S. Yu, J. W. G. Yarnell, P. M. Sweetnam, and L. Murray, "What level of physical activity protects against premature cardiovascular death? The Caerphilly study," Heart, vol. 89, no. 5, pp. 502-506, 2003.

[17] J. A. Levine, "Non-exercise activity thermogenesis (NEAT)," Best Practice \& Research Clinical Endocrinology \& Metabolism, vol. 16, no. 4, pp. 679-702, 2002.

[18] G. N. Healy, D. W. Dunstan, J. E. Shaw, P. Z. Zimmet, and N. Owen, "Objectively measured sedentary time and lightintensity physical activity are independently associated with components of the metabolic syndrome: the AusDiab study," Diabetologia, vol. 50, supplement 1, pp. S67-S68, 2007.

[19] R. K. Dishman and J. Buckworth, "Increasing physical activity: a quantitative synthesis," Medicine and Science in Sports and Exercise, vol. 28, no. 6, pp. 706-719, 1996.

[20] M. T. Hamilton, D. G. Hamilton, and T. W. Zderic, "Role of low energy expenditure and sitting in obesity, metabolic syndrome, type 2 diabetes, and cardiovascular disease," Diabetes, vol. 56, no. 11, pp. 2655-2667, 2007.

[21] A. Liberati, D. G. Altman, J. Tetzlaff et al., "The PRISMA statement for reporting systematic reviews and meta-analyses of studies that evaluate healthcare interventions: explanation and elaboration," British Medical Journal, vol. 339, Article ID b2700, 2009.

[22] B. E. Ainsworth, W. L. Haskell, M. C. Whitt et al., "Compendium of physical activities: an update of activity codes and MET intensities," Medicine and Science in Sports and Exercise, vol. 32, supplement 9, pp. S498-S504, 2000.

[23] S. H. Downs and N. Black, "The feasibility of creating a checklist for the assessment of the methodological quality both of randomised and non-randomised studies of health care interventions," Journal of Epidemiology and Community Health, vol. 52, no. 6, pp. 377-384, 1998.

[24] S. A. Newell, J. A. Bowman, and J. D. Cockburn, "Can compliance with nonpharmacologic treatments for cardiovascular disease be improved?" American Journal of Preventive Medicine, vol. 18, no. 3, pp. 253-261, 2000. 
[25] J. F. Sallis, J. J. Prochaska, and W. C. Taylor, "A review of correlates of physical activity of children and adolescents," Medicine and Science in Sports and Exercise, vol. 32, no. 5, pp. 963-975, 2000.

[26] C. Keller and R. P. Treviño, "Effects of two frequencies of walking on cardiovascular risk factor reduction in Mexican American women," Research in Nursing and Health, vol. 24, no. 5, pp. 390-401, 2001.

[27] K. L. Cox, V. Burke, A. R. Morton, L. J. Beilin, and I. B. Puddey, "The independent and combined effects of 16 weeks of vigorous exercise and energy restriction on body mass and composition in free-living overweight men-a randomized controlled trial," Metabolism: Clinical and Experimental, vol. 52, no. 1, pp. 107-115, 2003.

[28] K. L. Cox, V. Burke, A. R. Morton, L. J. Beilin, and I. B. Puddey, "Independent and additive effects of energy restriction and exercise on glucose and insulin concentrations in sedentary overweight men," The American Journal of Clinical Nutrition, vol. 80, no. 2, pp. 308-316, 2004.

[29] K. L. Cox, I. B. Puddey, A. R. Morton, V. Burke, L. J. Beilin, and M. McAleer, "Exercise and weight control in sedentary overweight men: effects on clinic and ambulatory blood pressure," Journal of Hypertension, vol. 14, no. 6, pp. 779-790, 1996.

[30] M. Krause, J. Rodrigues-Krause, C. O’Hagan et al., “The effects of aerobic exercise training at two different intensities in obesity and type 2 diabetes: implications for oxidative stress, low-grade inflammation and nitric oxide production," European Journal of Applied Physiology, vol. 114, no. 2, pp. 251-260, 2014.

[31] C. L. Goldie, C. A. Brown, S. M. J. Hains, J. L. Parlow, and R. Birtwhistle, "Synergistic effects of low-intensity exercise conditioning and $\beta$-blockade on cardiovascular and autonomic adaptation in pre- and postmenopausal women with hypertension," Biological Research for Nursing, vol. 15, no. 4, pp. 433-442, 2013.

[32] V. A. Cornelissen, J. Arnout, P. Holvoet, and R. H. Fagard, "Influence of exercise at lower and higher intensity on blood pressure and cardiovascular risk factors at older age," Journal of Hypertension, vol. 27, no. 4, pp. 753-762, 2009.

[33] M. H. Davenport, M. F. Mottola, R. McManus, and R. Gratton, "A walking intervention improves capillary glucose control in women with gestational diabetes mellitus: a pilot study," Applied Physiology, Nutrition, and Metabolism, vol. 33, no. 3, pp. 511-517, 2008.

[34] H. Allgayer, S. Nicolaus, and S. Schreiber, "Decreased interleukin-1 receptor antagonist response following moderate exercise in patients with colorectal carcinoma after primary treatment," Cancer Detection and Prevention, vol. 28, no. 3, pp. 208-213, 2004.

[35] A. A. Thorp, B. A. Kingwell, P. Sethi, L. Hammond, N. Owen, and D. W. Dunstan, "Alternating bouts of sitting and standing attenuate postprandial glucose responses," Medicine and Science in Sports and Exercise, vol. 46, no. 11, pp. 2053-2061, 2014.

[36] M. L. Mestek, E. P. Plaisance, L. A. Ratcliff, J. K. Taylor, S.-O. Wee, and P. W. Grandjean, "Aerobic exercise and postprandial lipemia in men with the metabolic syndrome," Medicine and Science in Sports and Exercise, vol. 40, no. 12, pp. 2105-2111, 2008.

[37] A. E. Mendham, C. E. Donges, E. A. Liberts, and R. Duffield, "Effects of mode and intensity on the acute exercise-induced IL6 and CRP responses in a sedentary, overweight population," European Journal of Applied Physiology, vol. 111, no. 6, pp. 10351045, 2011.
[38] D. W. Dunstan, B. A. Kingwell, R. Larsen et al., "Breaking up prolonged sitting reduces postprandial glucose and insulin responses," Diabetes Care, vol. 35, no. 5, pp. 976-983, 2012.

[39] R. N. Larsen, B. A. Kingwell, P. Sethi, E. Cerin, N. Owen, and D. W. Dunstan, "Breaking up prolonged sitting reduces resting blood pressure in overweight/obese adults," Nutrition, Metabolism and Cardiovascular Diseases, vol. 24, no. 9, pp. 976982, 2014.

[40] D. P. Bailey and C. D. Locke, "Breaking up prolonged sitting with light-intensity walking improves postprandial glycemia, but breaking up sitting with standing does not," Journal of Science and Medicine in Sport, vol. 18, no. 3, pp. 294-298, 2014.

[41] L. Skoro-Kondza, S. S. Tai, R. Gadelrab, D. Drincevic, and T. Greenhalgh, "Community based yoga classes for type 2 diabetes: an exploratory randomised controlled trial," $B M C$ Health Services Research, vol. 9, article 33, 8 pages, 2009.

[42] A. Gordon, R. Tyni-Lenné, H. Persson, L. Kaijser, E. Hultman, and C. Sylvén, "Markedly improved skeletal muscle function with local muscle training in patients with chronic heart failure," Clinical Cardiology, vol. 19, no. 7, pp. 568-574, 1996.

[43] W. D. Dudgeon, K. D. Phillips, J. L. Durstine et al., "Individual exercise sessions alter circulating hormones and cytokines in HIV-infected men," Applied Physiology, Nutrition, and Metabolism, vol. 35, no. 4, pp. 560-568, 2010.

[44] J. F. Horowitz, R. Mora-Rodriguez, L. O. Byerley, and E. F. Coyle, "Substrate metabolism when subjects are fed carbohydrate during exercise," The American Journal of PhysiologyEndocrinology and Metabolism, vol. 276, no. 5, pp. E828-E835, 1999.

[45] T. Fujimoto, J. Kemppainen, K. K. Kalliokoski, P. Nuutila, M. Ito, and J. Knuuti, "Skeletal muscle glucose uptake response to exercise in trained and untrained men," Medicine and Science in Sports and Exercise, vol. 35, no. 5, pp. 777-783, 2003.

[46] N. V. Tsetsonis and A. E. Hardman, "The influence of the intensity of treadmill walking upon changes in lipid and lipoprotein variables in healthy adults," European Journal of Applied Physiology and Occupational Physiology, vol. 70, no. 4, pp. 329-336, 1995.

[47] N. V. Tsetsonis, "Effects of low and moderate intensity treadmill walking on postprandial lipaemia in healthy young adults," European Journal of Applied Physiology and Occupational Physiology, vol. 73, no. 5, pp. 419-426, 1996.

[48] R. Perini, C. Orizio, A. Comande, M. Castellano, M. Beschi, and A. Veicsteinas, "Plasma norepinephrine and heart rate dynamics during recovery from submaximal exercise in man," European Journal of Applied Physiology and Occupational Physiology, vol. 58, no. 8, pp. 879-883, 1989.

[49] H. E. Aldred, I. C. Perry, and A. E. Hardman, "The effect of a single bout of brisk walking on postprandial lipemia in normolipidemic young adults," Metabolism, vol. 43, no. 7, pp. 836-841, 1994.

[50] H. E. Pay, A. E. Hardman, G. J. W. Jones, and A. Hudson, "The acute effects of low-intensity exercise on plasma lipids in endurance-trained and untrained young adults," European Journal of Applied Physiology and Occupational Physiology, vol. 64, no. 2, pp. 182-186, 1992.

[51] R. A. Hughes, W. G. Thorland, T. Eyford, and T. Hood, "The acute effects of exercise duration on serum lipoprotein metabolism," The Journal of Sports Medicine and Physical Fitness, vol. 30, no. 1, pp. 37-44, 1990.

[52] G. A. Wittert, D. E. Stewart, M. P. Graves et al., "Plasma corticotrophin releasing factor and vasopressin responses to 
exercise in normal man," Clinical Endocrinology, vol. 35, no. 4, pp. 311-317, 1991.

[53] C. Goto, Y. Higashi, M. Kimura et al., "Effect of different intensities of exercise on endothelium-dependent vasodilation in humans: role of endothelium-dependent nitric oxide and oxidative stress," Circulation, vol. 108, no. 5, pp. 530-535, 2003.

[54] I.-Y. Kim, S. Park, J. R. Trombold, and E. F. Coyle, "Effects of moderate- and intermittent low-intensity exercise on Postprandial Lipemia," Medicine and Science in Sports and Exercise, vol. 46, no. 10, pp. 1882-1890, 2014.

[55] R. Van Den Berg, S. De Groot, K. M. A. Swart, and L. H. V. Van Der Woude, "Physical capacity after 7 weeks of low-intensity wheelchair training," Disability \& Rehabilitation, vol. 32, no. 26, pp. 2244-2252, 2010.

[56] J. S. Stevenson and R. Topp, "Effects of moderate and low intensity long-term exercise by older adults," Research in Nursing \& Health, vol. 13, no. 4, pp. 209-218, 1990.

[57] B. M. F. M. Duvivier, N. C. Schaper, M. A. Bremers et al., "Minimal intensity physical activity (standing and walking) of longer duration improves insulin action and plasma lipids more than shorter periods of moderate to vigorous exercise (cycling) in sedentary subjects when energy expenditure is comparable," PLoS ONE, vol. 8, no. 2, Article ID e55542, 2013.

[58] American College of Sports Medicine, "Proper and improper weight loss programs," Medicine and Science in Sports and Exercise, vol. 15, no. 1, pp. 9-13, 1983.

[59] M. L. Stefanick, "Exercise and weight control," Exercise and Sport Sciences Reviews, vol. 21, pp. 363-396, 1993.

[60] J.-P. Despres and B. Lamarche, "Effects of diet and physical activity on adiposity and body fat distribution: implications for the prevention of cardiovascular disease," Nutrition Research Reviews, vol. 6, no. 1, pp. 137-159, 1993.

[61] J. W. Rankin, "Effective diet and exercise interventions to improve body composition in obese individuals," American Journal of Lifestyle Medicine, vol. 9, no. 1, pp. 48-62, 2015.

[62] H. Kohl and T. Murray, Foundations of Physical Activity and Public Health, Human Kinetics, 2012.

[63] C. S. Katsanos, P. W. Grandjean, and R. J. Moffatt, "Effects of low and moderate exercise intensity on postprandial lipemia and postheparin plasma lipoprotein lipase activity in physically active men," Journal of Applied Physiology, vol. 96, no. 1, pp. 181188, 2004.

[64] S. F. M. Chastin, T. Egerton, C. Leask, and E. Stamatakis, "Meta-analysis of the relationship between breaks in sedentary behavior and cardiometabolic health," Obesity, vol. 23, no. 9, pp. 1800-1810, 2015.

[65] J. Kim, K. Tanabe, N. Yokoyama, H. Zempo, and S. Kuno, "Objectively measured light-intensity lifestyle activity and sedentary time are independently associated with metabolic syndrome: a cross-sectional study of Japanese adults," International Journal of Behavioral Nutrition and Physical Activity, vol. 10, article 30, 2013.

[66] P. D. Loprinzi, H. Lee, and B. J. Cardinal, "Daily movement patterns and biological markers among adults in the United States," Preventive Medicine, vol. 60, pp. 128-130, 2014.

[67] R. H. Eckel, "Lipoprotein lipase: a multifunctional enzyme relevant to common metabolic diseases," The New England Journal of Medicine, vol. 320, no. 16, pp. 1060-1068, 1989.

[68] M. A. Kantor, E. M. Cullinane, P. N. Herbert, and P. D. Thompson, "Acute increase in lipoprotein lipase following prolonged exercise," Metabolism: Clinical and Experimental, vol. 33, no. 5, pp. 454-457, 1984.
[69] M. L. Pollock, G. A. Gaesser, J. D. Butcher et al., "The recommended quantity and quality of exercise for developing and maintaining cardiorespiratory and muscular fitness, and flexibility in healthy adults," Medicine and Science in Sports and Exercise, vol. 30, no. 6, pp. 975-991, 1998.

[70] J.-P. Despres and B. Lamarche, "Low-intensity endurance exercise training, plasma lipoproteins and the risk of coronary heart disease," Journal of Internal Medicine, vol. 236, no. 1, pp. 7-22, 1994.

[71] U. Ekelund, H. A. Ward, T. Norat et al., "Physical activity and allcause mortality across levels of overall and abdominal adiposity in European men and women: the European Prospective Investigation into Cancer and Nutrition Study (EPIC)," The American Journal of Clinical Nutrition, vol. 101, no. 3, pp. 613-621, 2015.

[72] G. E. Duncan, M. G. Perri, D. W. Theriaque, A. D. Hutson, R. H. Eckel, and P. W. Stacpoole, "Exercise training, without weight loss, increases insulin sensitivity and postheparin plasma lipase activity in previously sedentary adults," Diabetes Care, vol. 26, no. 3, pp. 557-562, 2003.

[73] G. N. Healy, E. A. Winkler, C. L. Brakenridge, M. M. Reeves, E. G. Eakin, and M. Alemany, "Accelerometer-derived sedentary and physical activity time in overweight/obese adults with type 2 diabetes: cross-sectional associations with cardiometabolic biomarkers," PLOS ONE, vol. 10, no. 3, Article ID e0119140, 2015.

[74] P. D. Loprinzi, H. Lee, and B. J. Cardinal, "Evidence to support including lifestyle light-intensity recommendations in physical activity guidelines for older adults," American Journal of Health Promotion, vol. 29, no. 5, pp. 277-284, 2015. 


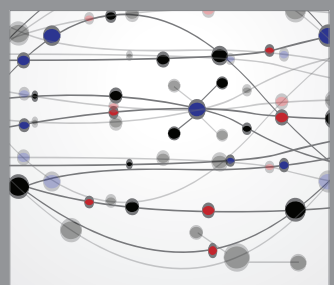

The Scientific World Journal
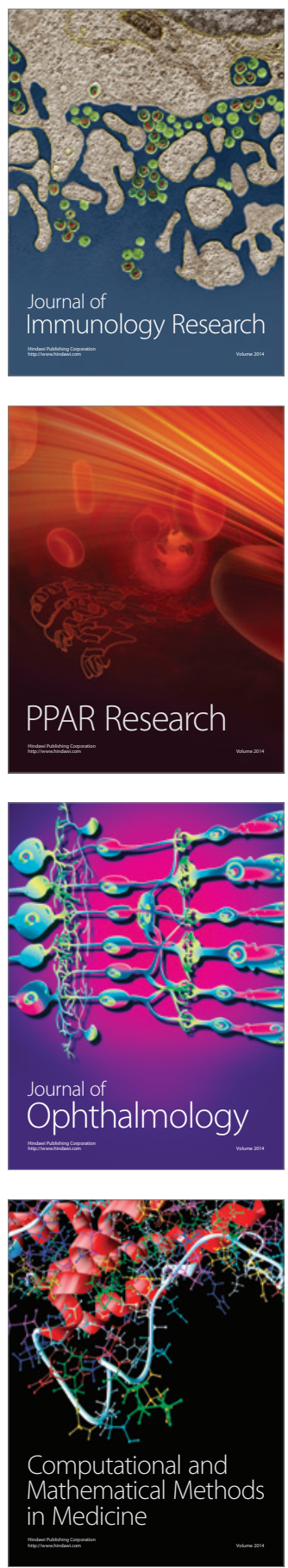

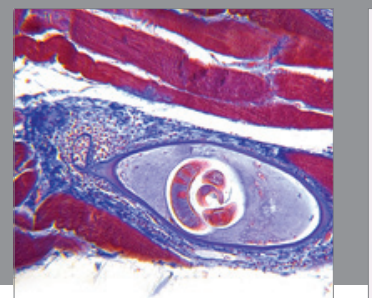

Gastroenterology

Research and Practice
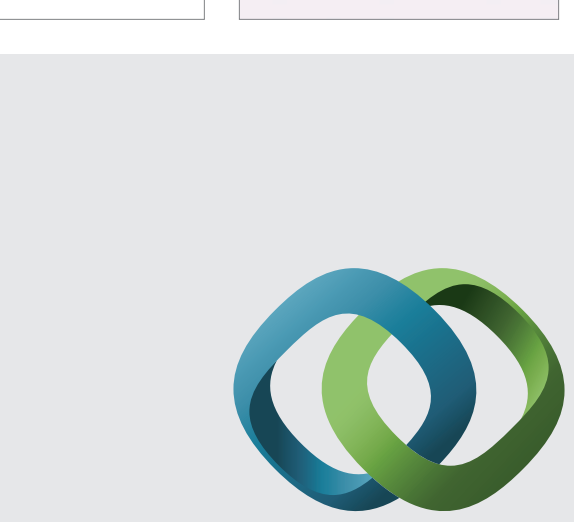

\section{Hindawi}

Submit your manuscripts at

http://www.hindawi.com
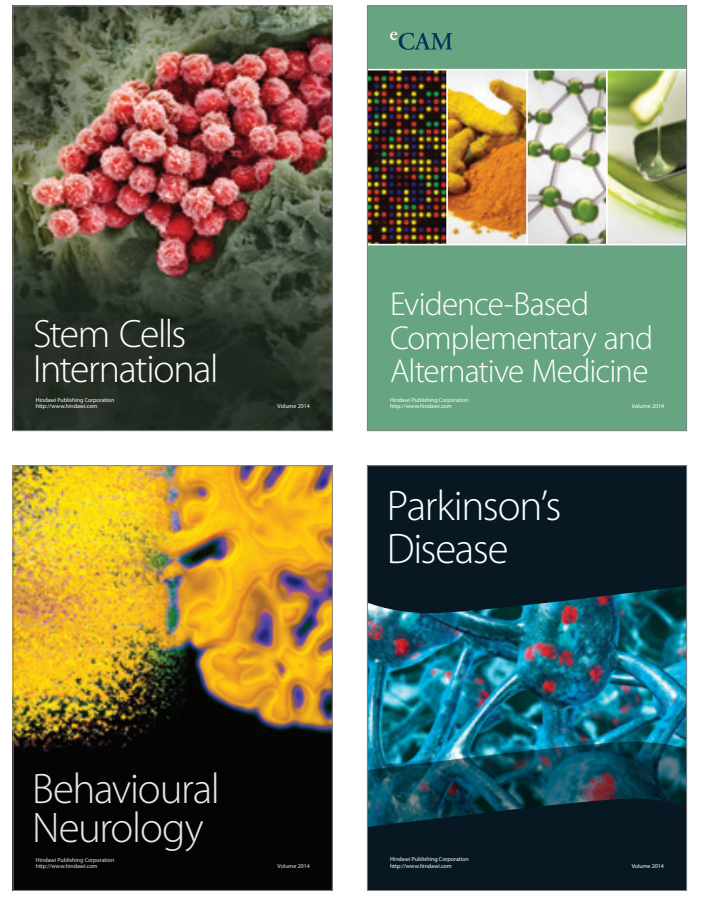
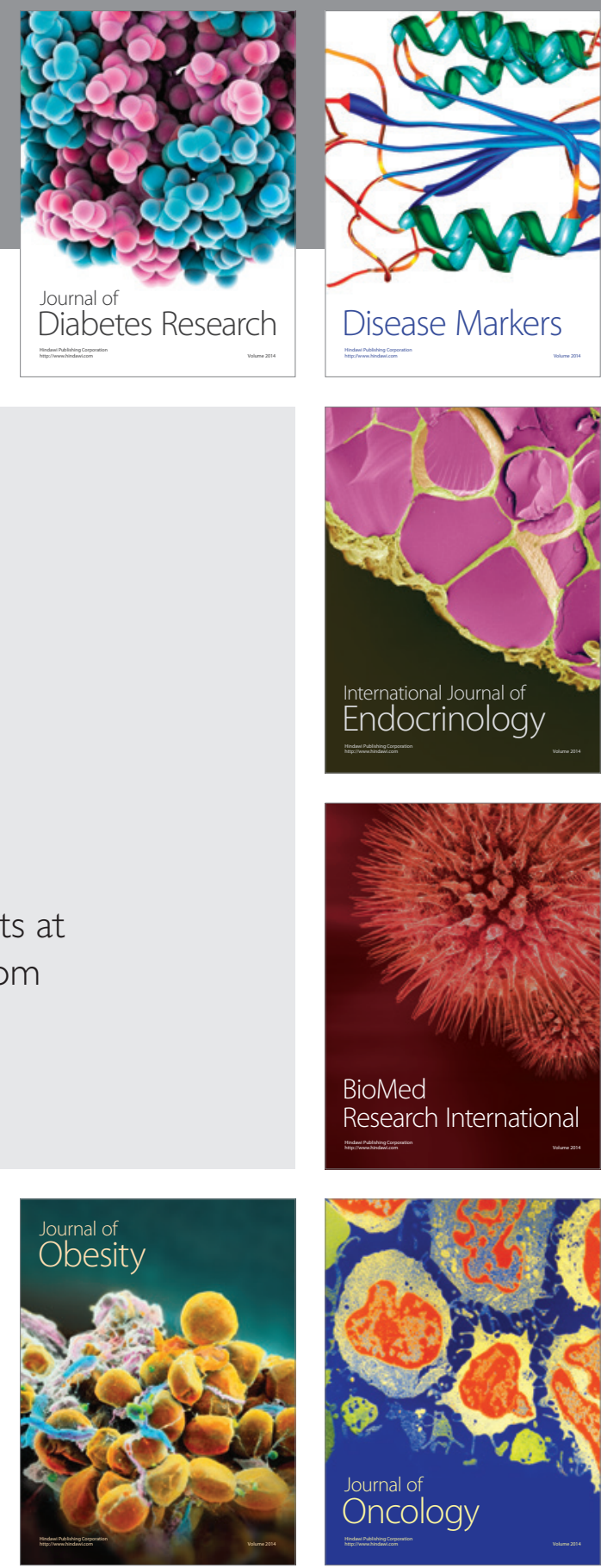

Disease Markers
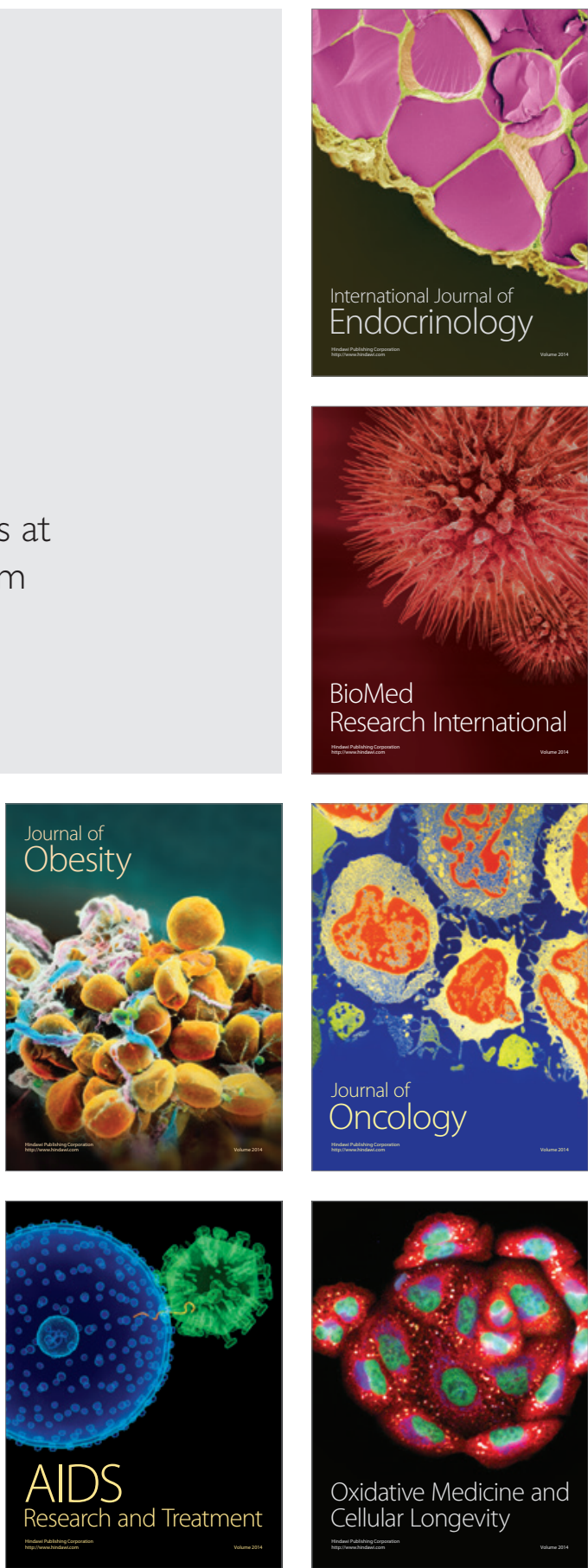